\title{
TECNOLOGIAS ASSISTIVAS: PROMOVENDO ACESSIBILIDADE DIGITAL NA EDUCAÇÃO
}

\section{ASSISTIVE TECHNOLOGIES: PROMOTING EDUCATION DIGITAL ACCESSIBILITY}

\author{
Rozimar Rodrigues de Brito ${ }^{1}$, Lic. \\ Adriano Patrício da Silva² ${ }^{2}$, Lic. \\ Mariano Castro Neto ${ }^{3}$, Dr. \\ (1) Universidade Federal da Paraíba (UFPB) \\ e-mail: rozimar.rodrigues@dcx.ufpb.br \\ (2) Universidade Federal de Campina Grande \\ e-mail: adrianopatricio@copin.ufcg.edu.br
(3) Universidade Federal da Paraíba
e-mail: castroneto.mariano@gmail.com

\section{Pessoas com deficiência, Educação, Acessibilidade}

\begin{abstract}
Este artigo apresenta as Tecnologias Assistivas como artefatos importantes à inclusão das pessoas com deficiências no contexto escolar. Este trabalho mostra alguns desses artefatos que podem possibilitar e proporcionar inclusão aos indivíduos com limitação, classificando em adaptações ou órteses, hardware e softwares. Por fim, sublinha-se que a utilização de Tecnologias Assistivas para apoio a aprendizagem realizados com pessoas com deficiências vem contribuindo de forma significativa para dinamizar conteúdos curriculares. Como continuação deste estudo e considerando a demanda de TA no que se referem à concepção, desenvolvimento, implementação e avaliação. É imperativo o desenvolvimento de artefatos para auxiliar a aprendizagem para pessoas com deficiência
\end{abstract}

People with Disabilities, Education, Accessibility.

This article presents the Assistive Technologies as important artifacts for promoting social inclusion of people with disabilities in the school contexto, and shows some of these artifacts that can enable and provide individuals inclusion with limitations, classifying adaptations or orthotics, hardware and software. Finally, the use of Assistive Technologies to support learning has contributed significantly to boosting curricular content for people with disabilities. Considering the demand for Assistive Technologies in terms of design, development, implementation and evaluation, the continuation of this study is imperative to develop artifacts to aid learning for people with disabilities. 


\section{$16^{\circ}$ \\ ERGODESIGN USIHC CINAHPA}

\section{Introdução}

$\mathrm{Na}$ atual conjuntura da sociedade observamos a predominância de uma grande diversidade de Tecnologias Assistivas (TA), e que a partir delas vislumbrados novas possibilidades e caminhos para de inclusão digital e social para pessoas com deficiências.

As TA surgem para auxiliar as pessoas com deficiências não apenas no contesto social, mas fundamentalmente no mundo da educação que por meio de softwares e hardwares educacionais, combinados proporcionam novas possibilidades de aprendizagens e descobertas, que sem os quais não seriam possíveis para algumas pessoas com deficiências com maior ou menor grau de severidade.

Em se tratando de acessibilidade digital Miranda e Zissou, afirmam que esse termo pode ser definido como a qualidade de ser acessível, o qual diz respeito a algo que é de fácil acesso e é inteligível, compreendido por várias pessoas independente de sua condição física ou cognitiva. [MIRANDA \& ZISSOU, 2009]

\section{Tecnologias Assistivas e Educação}

Do ponto de vista pedagógico Rita apresenta algumas diretrizes elaboradas por um grupo de pesquisadores da União Europeia consideradas por eles como sendo as mais apropriadas para a formação dos usuários finais de TA [BERSCH, 2008]:

Quadro 1 - Diretrizes para Formação

\begin{tabular}{|l|}
\hline Auxílios para a vida diária \\
\hline CCA(CSA) Comunicação aumentada e alternativa \\
\hline Recursos de acessibilidade ao computador \\
\hline Sistema de controle de ambiente \\
\hline Projetos arquitetônicos para acessibilidade \\
\hline Órteses e próteses \\
\hline Adequação postural \\
\hline Auxílios de mobilidade \\
\hline Auxílios para cegos ou com visão subnormal \\
\hline Auxílios para surdos ou com déficit auditivo \\
\hline Adaptações em veículos \\
\hline
\end{tabular}

Com base nessas diretrizes que são apresentadas no quadro 1 podemos observar que são vários $16^{\circ}$ Ergodesign - Congresso Internacional de Ergonomia e Usabilidade de Interfaces Humano Tecnológica: Produto, Informações Ambientes Construídos e Transporte

$16^{\circ}$ USIHC - Congresso Internacional de Ergonomia e Usabilidade de Interfaces Humano Computador

CINAHPA | 2017 - Congresso Internacional de Ambientes Hipermídia para Aprendizagem.

critérios que classificam os artefatos e que proporcionam acessibilidade. Também podemos observar que segundo essas diretrizes são muitas as possibilidades de desenvolvimento, criação ou adaptação desses artefatos na busca de desenvolver meios para melhor a aprendizagem e a inclusão das pessoas com deficiências.

\subsection{Educação Inclusiva}

Na educação inclusiva, identificam-se dois conceitos presentes no cotidiano escolar: integração e inclusão. A integração diz respeito à incorporação gradativa de alunos com deficiências em escolas regulares, podendo o aluno com deficiência permanecer parte do tempo na escola, em classes com recursos multifuncionais.

\subsubsection{Adaptações Físicas ou Órteses}

As adaptações físicas ou órteses são aparelhos ou adaptações desses aparelhos, que quando fixados no corpo do aluno deficiente proporciona assim uma facilidade maior na interação desses indivíduos com o computador. A pulseira de pesos reduz a amplitude do movimento que é causado pela flutuação no tônus, tornando o processo de digitação mais rápido e eficiente.

Figura 1 - Pulseiras de Peso

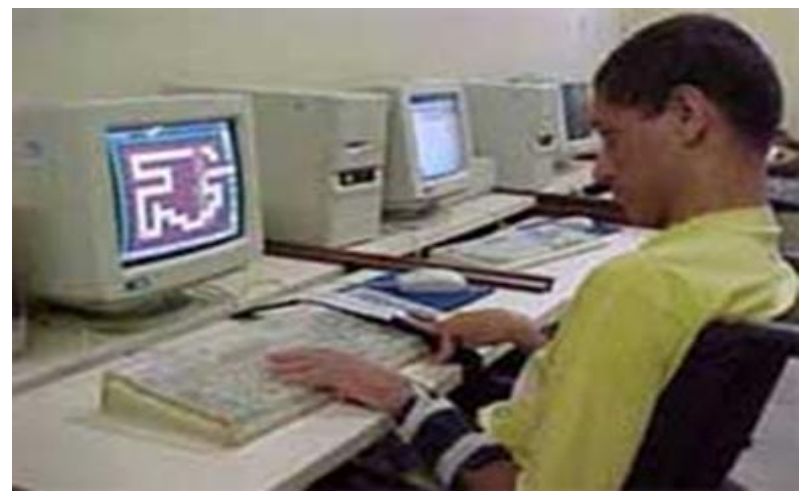

Realização:

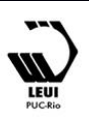




\section{$16^{\circ}$ \\ ERGODESIGN USIHC CINAHPA}

$16^{\circ}$ Ergodesign - Congresso Internacional de Ergonomia e Usabilidade de Interfaces Humano Tecnológica: Produto, Informações Ambientes Construídos e Transporte

$16^{\circ}$ USIHC - Congresso Internacional de Ergonomia e Usabilidade de Interfaces Humano Computador

CINAHPA | 2017 - Congresso Internacional de Ambientes Hipermídia para Aprendizagem.
Figura 2 - Utilização da pulseira de peso

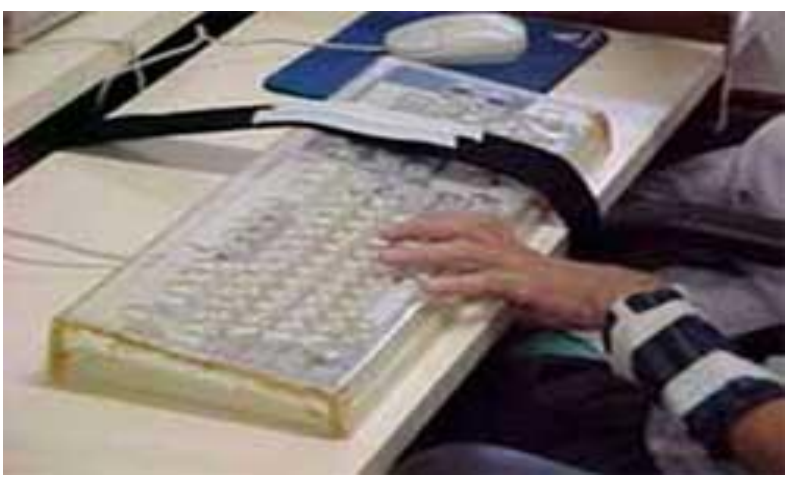

Outra órtese existente é o estabilizador de punho e abdutor de polegar com ponteira para digitação, para alunos, principalmente com paralisia cerebral que costumam apresentar essas necessidades. Com esse equipamento o deficiente consegue uma maior facilidade no desempenho diante do computador.

Figura 3 - Estabilizador de Punho e Abdutor de Polegar

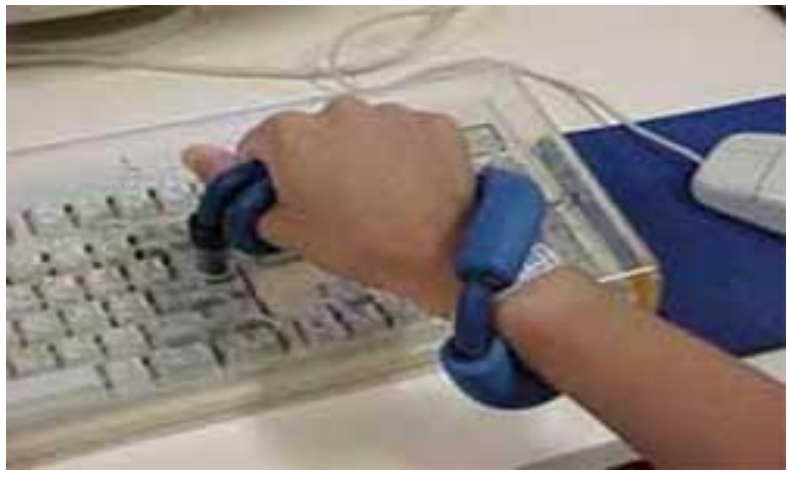

Além dessas adaptações físicas e órteses que podem ser utilizadas, existem várias outras que também podem ser úteis, dependendo das necessidades específicas de cada aluno, como os ponteiros de cabeça, ou hastes fixadas na boca ou queixo, quando existe o controle da cabeça, entre outras.
Figura 4 - Haste Fixada na Cabeça para Digitação

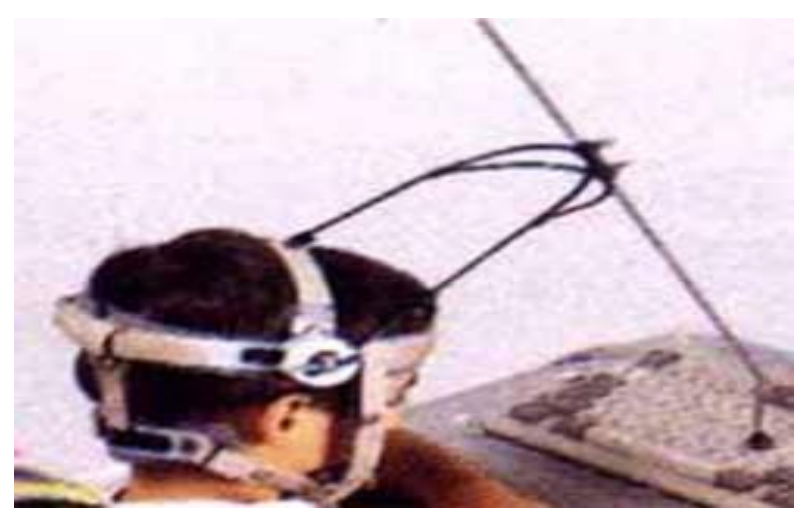

\section{Hardwares Usados na Educação Inclusiva}

Os hardwares também podem ser utilizados na educação inclusiva com adaptações feitas para melhorar o desempenho de alunos com limitações na utilização dos computadores um dos recursos mais simples de Hardware é a máscara de teclado ou colmeia.

Figura 5 - Máscara de Teclado Sobreposta

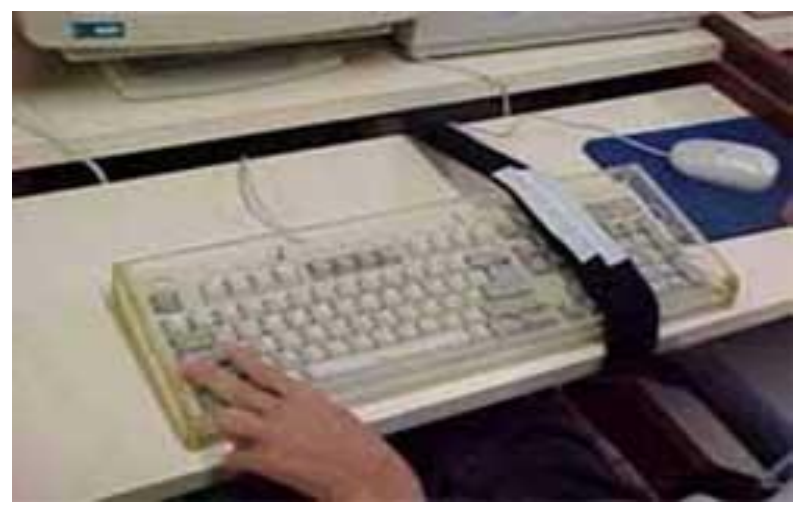




\section{$16^{\circ}$ \\ ERGODESIGN USIHC CINAHPA}

Figura 6 - Máscara de Teclado Encaixada

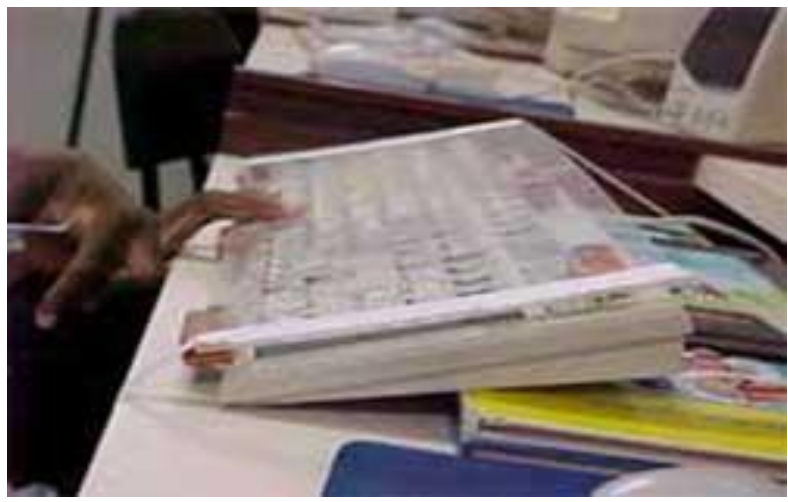

$\mathrm{O}$ aluno que possui deficiência mental e por isso tem problema de coordenação motora, podem utilizar a máscara de teclado juntamente com tampões de cartolina ou papelão, e deixar amostra somente as teclas que serão necessárias para utilizar enquanto estiverem realizando o trabalho.

Figura 7 - Teclado com Alteração na Inclinação e Fixado à Mesa

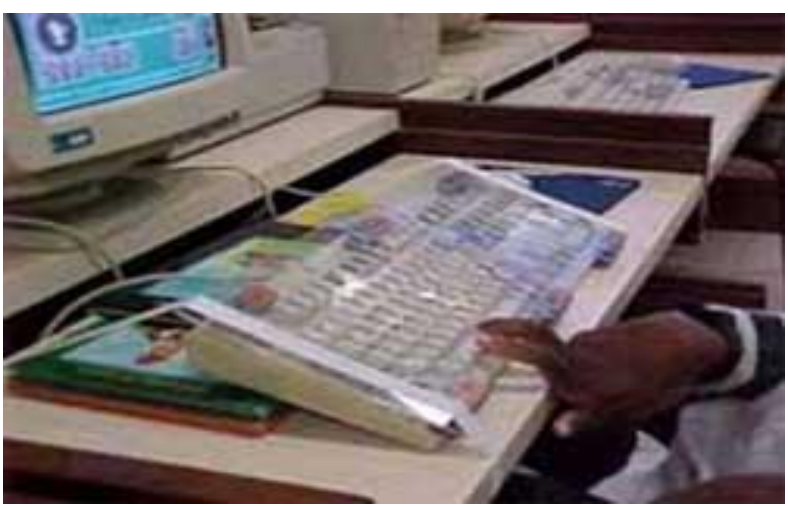

Além do posicionamento de hardwares no colo ou sobre um apoio, podem existir alunos que não possuam movimento nos braços e dessa forma tenham uma maior facilidade em trabalhar se o teclado estiver posicionado no chão ao alcance dos pês. $16^{\circ}$ Ergodesign - Congresso Internacional de Ergonomia e Usabilidade de Interfaces Humano Tecnológica: Produto, Informações Ambientes Construídos e Transporte

$16^{\circ}$ USIHC - Congresso Internacional de Ergonomia e Usabilidade de Interfaces Humano Computador

CINAHPA | 2017 - Congresso Internacional de Ambientes Hipermídia para Aprendizagem.
Figura 8 - Teclado reposicionado para digitação com o pé

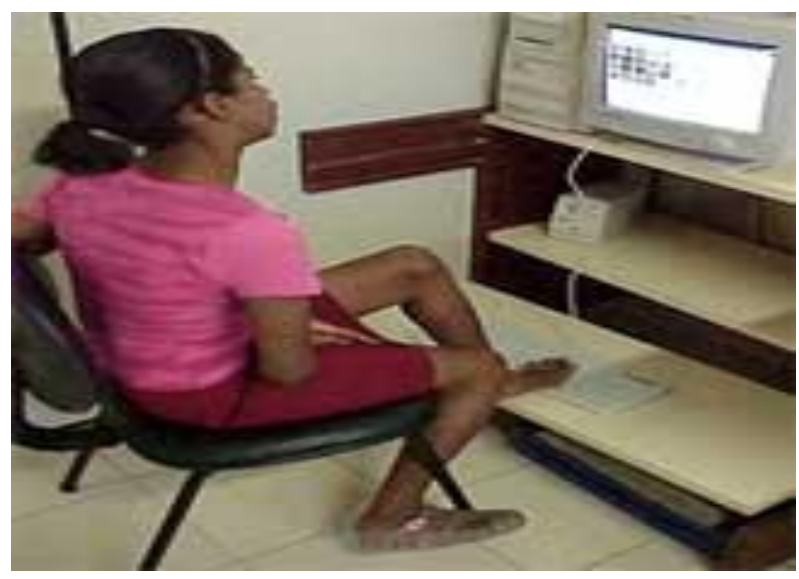

Além dos hardwares adaptados que mostramos aqui, existem vários outro que podem ser encontrados ou adaptações que podem ser feitas, a depender da necessidade e da observação do professor em procurar a melhor maneira para trabalhar com o aluno deficiente.

\subsubsection{Softwares Usados na Educação Inclusiva}

Os softwares assim como os hardwares são grandes aliados dos professores e dos próprios alunos no processo de inclusão e acessibilidade. Os softwares também podem ser voltados para uma determinada deficiência, onde os desenvolvedores procuram chegar a um produto que atenda o maior número de usuários com uma deficiência especifica.

\subsubsection{Hand Talk}

Ronaldo Tenório, Carlos Wanderlan e Thadeu Luz desenvolveram o Hand Talk, um aplicativo tradutor automático para LIBRAS para smartphones e tablets, android ou IOS, que quebra a barreira entre surdos e ouvintes. Foi desenvolvido em parceria com a Universidade Federal de Alagoas em um projeto de pesquisa que já possui mais de 10 anos de estudos em Tecnologia Assistida em LIBRAS. [HAND TALK, 2014] 
$16^{\circ}$ Ergodesign - Congresso Internacional de Ergonomia e Usabilidade de Interfaces Humano Tecnológica: Produto, Informações Ambientes Construídos e Transporte

$16^{\circ}$ USIHC - Congresso Internacional de Ergonomia e Usabilidade de Interfaces Humano Computador

CINAHPA | 2017 - Congresso Internacional de Ambientes Hipermídia para Aprendizagem.

\subsubsection{Dream Voice Reader}

O Voice Dream Reader é o único aplicativo capaz de ler em voz alta: artigos, documentos e livros. Está disponível apenas para IOS na Apple Store. Pode ser baixado gratuitamente no iTunes.

[ITUNES, 2017]

\subsubsection{Argot}

O Argot é um software para ensino de inglês, voltado para pessoas com Síndrome de Down. Na interface do sistema as figuras e palavras em maior tamanho facilitam a leitura, a identificação dos elementos da interface e consequentemente a interação do usuário com o sistema. Nas atividades, o plano de fundo é uma imagem de uma paisagem, representada por um campo em tons de verde e o céu azul, conforme figura 13. A distribuição dos elementos na interface necessita de equilíbrio. [FRANCISCATO e CANAL, 2007].

\subsubsection{Que-fala!}

O Que Fala! é uma solução para tablets e smartphones que possibilita a comunicação de pessoas com deficiência. Trata-se de uma prancha digital que pode substituir as pranchas de papel geralmente empregadas no tratamento de pacientes com dificuldades na fala.

\section{Conclusão}

O desenvolvimento deste estudo permitiu problematizar algumas considerações relevantes acerca da importância da utilização de TA em processos ensino-aprendizagem em uma perspectiva inclusiva. Nessa perspectiva observamos a grande variedade de TA para auxiliar pessoas com deficiência em processos ensinoaprendizagem, sejam em adaptações, hardwares ou softwares, que possibilitem acessibilidade aos seus usuários.

Apesar dessa gama de artefatos percebemos que existem barreiras a serem ultrapassadas uma vez que são vários os tipos de deficiências que se apresentam variando de indivíduo para indivíduo, e principalmente quando consideramos que cada indivíduo tem diferentes formas de aprender. Implicando em pesquisas multidisciplinares e com isso se complexificam.

Dentre as contribuições deste estudo, destacam-se:

a) No contexto educacional há dificuldades por parte dos professores na utilização de tecnologias Assistivas.

b) Reconhecer que a comunicação com o aluno deficiente é importante nesse processo e que há escolas que não possuem se quer interpretes de libras, por exemplo.

Por fim, sublinha-se que a utilização de Tecnologias Assistivas para auxiliar processos ensino-aprendizagem realizados com pessoas com deficiências vem contribuindo de forma significativa para dinamizar conteúdos curriculares. Como continuação deste estudo percebe-se que a TA ainda é um campo a ser explorado, e que há possibilidades de catalogar e desenvolver artefatos para auxiliar processos ensino-aprendizagem para pessoas com deficiência.

\section{BIBLIOGRAFIA}

BERSCH, Rita. Introdução à Tecnologia

Assistiva. CEDI - Centro Especializado em

Desenvolvimento Infantil. Porto Alegre, 2008.

FRANCISCATO, F. T.; CANAL, A. P. ArgotSoftware para ensino de inglês para pessoas com Síndrome de Down. In: Brazilian Symposium on Computers in Education (Simpósio Brasileiro de Informática na Educação-SBIE). 2007. p. 30-33.

HAND TALK. Hand Talk: Criatividade e Inovação da Molecada Brasileira.2014. Disponível em: <http://blog.isocial.com.br/hand-talkcriatividade-e-inovacao-da-molecada-brasileira/>. Acesso em: 18 mar. 2017.

ITUNES. Voice Dream Reader. Disponível em: <https://itunes.apple.com/br/app/voice-dreamreader/id496177674?mt=8\&>. Acesso em: 18 mar.
Realização:

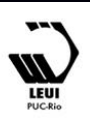




\section{$16^{\circ}$ \\ ERGODESIGN USIHC CINAHPA}

$16^{\circ}$ Ergodesign - Congresso Internacional de Ergonomia e Usabilidade de Interfaces Humano Tecnológica: Produto, Informações Ambientes Construídos e Transporte

$16^{\circ}$ USIHC - Congresso Internacional de Ergonomia e Usabilidade de Interfaces Humano Computador

CINAHPA | 2017 - Congresso Internacional de Ambientes Hipermídia para Aprendizagem.

2017.

MIRANDA, A. S.; ZISSOU, A. de J.

Considerações sobre Acessibilidade e

Usabilidade em Ambientes Hipermídia. In:

ULBRICHT, V. R.; PEREIRA, A. T. C.

Hipermídia: um desafio da atualidade.

Florianópolis: Pandion, 2009. p.16-29. 